\title{
Verkörperte Räume - «verräumte» Körper Zu einem feministisch-poststrukturalistischen Verständnis der Wechselwirkungen von Körper und Raum
}

\author{
Sybille Bauriedl, Hamburg, Katharina Fleischmann, \\ Berlin, Anke Strüver, Nijmegen, Claudia Wucherpfen- \\ nig, Frankfurt am Main
}

Angesichts globaler Restrukturierungsprozesse ist die Geographie mit neuen gesellschaftlichen Phänomenen konfrontiert, die (auch) eine Herausforderung an die Theoriebildung darstellen.

Da das gesellschaftskritische Potential poststrukturalistischer Theorien oftmals schwer zu fassen ist, haben wir versucht, diesen Ansatz zunächst mit Metaphern zu umschreiben:

\section{Der Körper in der Bergbahn zwischen Bahnhof Zoo und Zugspitze}

Start unserer Reise ist der Bahnhof Zoo in Berlin. Auf dem Weg zum Gleis riechen wir etwas Süßes; ein verführerischer Duft kitzelt uns in der Nase und führt uns direkt zum Body Shop. Dort steht in großen Lettern geschrieben: «WIEDER DA! POSTSTRUKTURALISMUS ZUM SCHNUPPER-PREIS!»

Wir greifen zu und packen den schweren Poststrukturalismus in unseren Koffer voller Worte. Die Lust wird zur Last. Wir schleppen uns und den Koffer zum Zug und entscheiden uns für einen Platz an der Zugspitze. Unsere Mägen melden sich zu Wort, wir haben Hunger. Irgendwo im Koffer muß doch unser Reiseproviant und die Nervennahrung sein? Wir öffnen den Koffer: Der Poststrukturalismus hat mittlerweile die Worte im Koffer dekonstruiert und die Bedeutung ist dabei verloren gegangen. Hat er etwa auch den BrotLaib angegriffen und entmaterialisiert?

Zum Teil, aber er hat sich am harten Knust ganz schön die Zähne ausgebissen.

Trotz der veränderten Zustände im KofferRaum müssen wir zunächst etwas essen, denn der Magen knurrt so laut, daß unsere Worte nicht mehr $\mathrm{zu}$ hören sind. Nachdem wir die körperlichen Bedürfnisse befriedigt haben, stellen wir uns erneut den Realitäten im Koffer:

Was hat der Poststrukturalismus mit dem Leib geMacht?

Was ist mit dem Raum passiert?

Wo ist der Sinn?
Die Frage - bestenfalls - nach dem Sinn haben sich im Mai 1999 viele, nicht nur Geographen und Geographinnen, gestellt. Zu dieser Zeit erschien der Titel unseres Vortrags «Der Körper in der Bergbahn zwischen Bahnhof Zoo und Zugspitze», den wir beim 52. Deutschen Geographentag in Hamburg präsentiert haben, im Nachrichtenmagazin «Der Spiegel» in der Rubrik «Hohlspiegel». Durch dieses verwirrende Spiel mit Worten und Bedeutungen wollten wir die Ernsthaftigkeit unseres inhaltlichen Anliegens allerdings nicht untergraben. Vielmehr geht es darum, ob eine poststrukturalistische Körperbetrachtung für eine Raumwissenschaft Sinn macht.

Dieser Beitrag thematisiert nun die Konstitutionsbedingungen von menschlichen Körpern und Räumen sowie deren dynamisches Wechselverhältnis. Der metaphorisch gedachte Obertitel zielt dabei nicht auf die Erörterung eines spezifischen Aspekts, z.B. den der Raumüberwindung, sondern dient der Verdeutlichung des dynamischen Verhältnisses von Körpern und Räumen, wenn auch auf eine persiflierende Art.

Menschliche Körper, oftmals auch Räume, werden in der Geographie als etwas Offen-Sichtliches und SelbstVerständliches betrachtet.

Wie wir uns geben und wie wir uns und andere sehen, ist jedoch nicht naturgegeben, sondern durch gesellschaftliche Prozesse beeinflußt. Auch Körper sind gesellschaftlich konstruiert und stehen in einem räumlichen Kontext. Es macht daher wenig Sinn, Körper ohne Raum oder Raum ohne unterschiedliche Körper zu betrachten. Während sich um die Bedeutung bzw. den Bedeutungsverlust des Raumes Wissenschaftler und Wissenschaftlerinnen vieler Disziplinen streiten, wurde der Körper im geographischen Alltag bisher kaum thematisiert.

Um mit dem Körper in der Geographie voranzukommen, unternehmen wir die folgende Reise mit vier Haltepunkten: An unserem ersten Haltepunkt betrachten wir den theoretischen Hintergrund von Körper- und Raumkonstruktionen. Der zweite Stop verdeutlicht, wie Körper von außen wahrgenommen werden. Der nächste Halt läßt uns einen Blick auf die körperbezogene Eigenwahrnehmung verschiedener Menschen werfen. Geschärft wird dieser Blick durch das Beispiel von Frauen mit Brustkrebs. Ziel der Fahrt ist es, Fremd- und Eigenwahrnehmung in ihrer Bedeutung für die vielfältigen Raumaneignungsstrategien zu begreifen. 
Nähern werden wir uns nun den Wechselverhältnissen von Körpern und Räumen aus feministisch-poststrukturalistischer Perspektive. Diese eignet sich unseres Erachtens besonders, um die Konstitutionsbedingungen sowohl von Körpern als auch von Räumen erfassen und hinterfragen zu können. Zudem möchten wir damit einen theoretischen Ansatz zur Diskussion stellen, der in der deutschsprachigen Geographie bisher nur vereinzelt aufgegriffen worden ist.

\section{Lokal verankerte Körper}

Unter Poststrukturalismus werden verschiedene theoretische Positionen gefaßt, die die Analyse gesellschaftlicher Strukturen mit der Ebene der individuellen Erfahrung verbinden. Ziel dieser Ansätze ist die Entwicklung von Strategien zum Aufbrechen hierarchischer Strukturen und Mechanismen. Es handelt sich um Theorien, die die Beziehungen zwischen Gesellschaftsordnung, Subjektivität und Macht thematisieren. Dabei wird nicht von einem Subjekt im humanistischen Verständnis ausgegangen, sondern von einem durch Sprache und gesellschaftliche Praktiken konstruierten, von einem Subjekt, das Ausdruck der individuellen Identifikationsprozesse innerhalb der herrschenden Gesellschaftsordnung ist (WEEDON 1990).

Zentral ist in den poststrukturalistischen Ansätzen die Dekonstruktion. Darunter wird im allgemeinen die Infragestellung aller essentialistischen und normierenden Kategorien und des Anspruches auf Wahrheit, Objektivität und Universalität verstanden. Ziel der Dekonstruktion ist es, die Herstellung und Wirkung von verschiedenen, z.T. einander widersprechenden Bedeutungen zu erfassen und gesellschaftliche Machtstrukturen aufzudecken. Zudem verfolgt sie die Erläuterung und Anerkennung von Differenzen. Und zwar nicht im Sinne einer Auflösung von Gegensätzen, sondern verstanden als Respektierung der vielfältigen Lebensrealitäten (DERrIDA 1976).

Der feministische Poststrukturalismus konzentriert sich auf die Denaturalisierung der Geschlechterdifferenz und damit auf die Konstitutionsprozesse von Körper und Geschlecht. Auf der Ebene der hegemonialen gesellschaftlichen Machtverhältnisse werden die Mechanismen aufgezeigt, die Subjekte durch Einund Ausschluß, durch vermeintlich grundlegende, natürlich-biologische Unterschiede konstruieren.

Grundlegende gesellschaftliche Strukturkategorien wie Ethnizität, Geschlecht oder Alter, anhand derer Individuen und soziale Gruppen klassifiziert werden und ihnen ein Platz in unserer Gesellschaft zugewiesen wird, rekurrieren direkt auf äußerliche körperliche Merkmale.
In hegemonialen Diskursen, die in Institutionen und gesellschaftlichen Praktiken verankert sind und durch die unentwegt Gesellschaftsstrukturen (re)produziert werden, wird der Körper zu einer Grundlage von Zuordnungen, Kategorisierungen und Hierarchisierungen. Subjekte werden reduktionistisch über bestimmte Körpermerkmale wahrgenommen oder gar als Körper konstituiert: die Frau, der Schwarze, die Alte usw. Solche Kategorien und ihre körperlichen «Grundlagen» werden in der Regel als universell und natürlich betrachtet.

Aus (feministisch-)poststrukturalistischer Sicht sind sie jedoch keine Grundlagen, sondern Effekte gesellschaftlicher Machtverhältnisse: Ein poststrukturalistisches Körperverständnis wirkt der Auffassung von der «natürlich-gegebenen» Normativität des Körpers entgegen: Menschliche Körper werden als materielle Verkörperung gesellschaftlicher Strukturen und Prozesse verstanden, d.h. soziale Prozesse werden individuell verinnerlicht, sozusagen «einverleibt», und dadurch verkörpert. Körperliche Erfahrungen basieren nicht auf durch das Wesen der Menschen vorgegebenen, natürlich-subjektiven Empfindungen, sondern stellen auch diskursiv erzeugte Effekte gesellschaftlich dominanter Werte und Normen dar:

«Die sozial vorgegebenen Körpercodes haben die Macht, die je eigenen Körpererfahrungen zu besetzen, zu kolonisieren, sich dem Leib einzuschreiben» (List 1994:19).

Oder in den Worten Judith ButLers (1997):

«Der Körper ist nicht Basis, sondern materialisierter Effekt sozialer Prozesse.»

Und auch Michel Foucault (1978) hat sich diesbezüglich geäußert:

«Der Körper wird durch die Machtverhältnisse als Subjekt konstituiert, er ist kein Gegenüber der Macht, sondern eine ihrer ersten Wirkungen.»

Derartige Aussagen klären zwar das «was», nicht aber das «wie». Zu fragen ist also, durch welche Praktiken Körper konstruiert und Bedeutungen inszeniert werden.

Judith Butler $(1997,1998)$ hat dargelegt, daß die Sprache als Bezeichnungs- und Bedeutungspraxis bei der Konstruktion von Körpern und Subjekten eine fundamentale Rolle spielt:

«Sprache erhält den Körper nicht, indem sie ihn im wörtlichen Sinne ins Dasein bringt oder ernährt. Vielmehr wird eine bestimmte gesellschaftliche Existenz des Körpers erst dadurch ermöglicht, daß er sprachlich angerufen wird» (Butler 1998:14).

Diese Anrufung nimmt in körperlichen Stilen Form an und ist somit konstitutiv für die individuelle Subjektwerdung. Durch den Bezeichnungsprozeß entsteht der Effekt des Natürlichen; die Bezeichnung erzeugt den Effekt des Apriori des Bezeichneten. Das heißt, wenn ich mit einem eindeutig weiblichen Vornamen immer 
wieder direkt angeredet werde, konstituiert sich mein Körper als ein weiblicher und ich bin - bewußt oder unbewußt - gezwungen, mich einem der geschlechtlichen Prototypen «Mann» oder «Frau» zuzuordnen.

Im Proze $\beta$ der Subjektkonstitution durch Benennung und Anrufung werden damit auch Gesellschaftsstrukturen einverleibt. Pierre Bourdieu (1982) betont die fundamentale Bedeutung des Körpers als Ort der Inkorporierung sozialer Strukturen. Die gesellschaftliche Vorstellung des eigenen Körpers (Eigenwahrnehmung), die bei der Identitätsbildung als subjektives Bild vom je eigenen Körper konstitutiv eingeht, wird Bourdieu zufolge durch die Anwendung eines sozialen Klassifikationssystems erreicht. Dessen hierarchische Prinzipien unterscheiden sich in nichts von dem der gesellschaftlichen Produkte, auf die es angewendet wird.

Dieses soziale Klassifikationssystem wirkt auch konstitutiv auf Räume. Es läßt sich eine Ähnlichkeit der Konstruktionsmechanismen feststellen, die wir anhand der folgenden Gegenüberstellung verdeutlichen wollen:
Verkörperte Subjekte und Räume werden aber nicht unabhängig voneinander konstituiert, sondern stehen in einem unmittelbaren Zusammenhang. Das heißt, daß sowohl Raumstrukturen (wie z.B. die Trennung in öffentliche und private Räume) als auch unterschiedliche Körper mit spezifischen Rollenzuschreibungen sich gegenseitig produzieren und die dominanten Strukturen reproduzieren, wie wir im weiteren darlegen werden.

Wie in Tab. 1 aufgezeigt, erfolgt die Konstituierung von Subjekten und Subjektpositionen wie auch von Räumen auf der Ebene der Eigenwahrnehmung und auf der der Fremdwahrnehmung in einem Proze $ß$ der Naturalisierung (SPUNK 1999). Verbunden ist dies mit Diskriminierung und Ausgrenzung, was nachfolgend mittels einer analytischen Trennung ausgeführt wird. Die diskursive Konstruktion von Körpern und Räumen wirkt sich grundlegend auf die Eigenwahrnehmung, d.h. auf das eigene Verständnis des Selbst, des eigenen Körpers und die Räume aus, in denen sich dieser Körper (nicht) bewegt. Zugleich prägt sie die Wahr-

\begin{tabular}{|c|c|}
\hline Körper & Raum \\
\hline $\begin{array}{l}\text { Körper werden im allgemeinen als etwas Natürliches } \\
\text { und biologisch-organisch Vorgegebenes betrachtet. }\end{array}$ & $\begin{array}{l}\text { Raum wird oftmals als physisch-materielle Substanz } \\
\text { betrachtet, die als Grundlage für ökonomische, } \\
\text { kulturelle und soziale Nutzung dient. }\end{array}$ \\
\hline $\begin{array}{l}\text { Körper sind materielles Abbild gesellschaftlicher } \\
\text { Strukturen und Prozesse. }\end{array}$ & $\begin{array}{l}\text { Räume sind materielles Abbild gesellschaftlicher } \\
\text { Strukturen und Prozesse. }\end{array}$ \\
\hline $\begin{array}{l}\text { Gesellschaftliche Verhältnisse werden (in den Körper) } \\
\text { einverLEIBt (LIST 1994). }\end{array}$ & $\begin{array}{l}\text { Gesellschaftliche Verhältnisse werden in den Raum } \\
\text { eingeschrieben (BOURDIEU 1991). }\end{array}$ \\
\hline $\begin{array}{l}\text { Der Körper ist Austragungsort gesellschaftlicher } \\
\text { Machtverhältnisse (BOURDIEU 1982). }\end{array}$ & $\begin{array}{l}\text { Der Raum ist Austragungsort gesellschaftlicher } \\
\text { Machtverhältnisse (BOURDIEU 1991). }\end{array}$ \\
\hline $\begin{array}{l}\text { Körper sind materialisierte Effekte gesellschaftlicher } \\
\text { Diskurse und Machtformationen (BUTLER 1997). }\end{array}$ & $\begin{array}{l}\text { Räume sind materialisierte Effekte gesellschaftlicher } \\
\text { Diskurse und Machtformationen (BOURDIEU 1991). }\end{array}$ \\
\hline $\begin{array}{l}\text { Körper sind etwas Prozeßhaftes, ein Prozeß, in dem die } \\
\text { regulierenden Normen den Körper in Form von } \\
\text { Wiederholungen und Variationen materialisieren } \\
\text { (BUTLER 1997). }\end{array}$ & $\begin{array}{l}\text { Räume sind etwas Prozeßhaftes, ein Prozeß, in dem die } \\
\text { regulierenden Normen den Raum in Form von } \\
\text { Wiederholungen und Variationen materialisieren } \\
\text { (BouRDiEu 1991). }\end{array}$ \\
\hline $\begin{array}{l}\text { Körper erweisen sich als Zeichen, die Bedeutungen } \\
\text { inszenieren und Wirklichkeit konstituieren (BUTLER } \\
\text { 1997). }\end{array}$ & $\begin{array}{l}\text { Räume erweisen sich als Zeichen, die Bedeutungen } \\
\text { inszenieren und Wirklichkeit konstituieren (BOURDIEU } \\
\text { 1991). }\end{array}$ \\
\hline
\end{tabular}

Tab. 1: Konstituierung von Körpern und Räumen

Constitution of bodies and spaces

Constitution des corps et des espaces 
nehmung aller anderen offen-sichtbaren Körper und Räume sowie deren Bedeutungszuschreibungen und Bewertungen. Werfen wir zunächst einen Blick auf die Fremdwahrnehmung:

\section{Offen-Sichtliche Körper: Fremdwahrnehmungen}

Im Alltag nehmen wir Räume und Menschen interpretierend wahr, wobei wir uns gesellschaftlicher Codes bedienen. Bestimmte äußerliche Merkmale dienen dabei als Zeichen, mittels derer Bedeutungen zugeschrieben werden und Wirklichkeit konstituiert wird.

Ein Beispiel kann solche Bedeutungszuschreibungen verdeutlichen: Ein ungepflegt erscheinender Mensch in alter, verschmutzter oder gar zerlumpter Kleidung und mit ungewaschenem Haar wird - unabhängig vom Realitätsgehalt - schnell als Obdachloser klassifiziert, die entsprechenden Attribute werden sogleich mitgedacht. Zweifellos unzweideutig ist auch sofort, daß er auf die Straße, unter eine Brücke oder auf eine Parkbank gehört - nicht aber etwa in eine Bank, in ein vornehmes Restaurant oder zu einem im Park stehenden Kinderwagen.

Solch ein überzeichnetes Beispiel birgt immer die Gefahr in sich, Zuschreibungen zu reproduzieren und zu zementieren. Es kann aber auch den Blick auf gesellschaftliche Realitäten schärfen und, wie es der Schriftsteller Wilhelm Genazino (1999) ausgedrückt hat, auf eine «peinliche Eigentümlichkeit unseres Bewußtseins hinweisen: Es identifiziert, bevor es denkt.»

Verallgemeinert ausgedrückt bedeutet dies: Anhand körperlicher Merkmale, wie etwa der Hautfarbe, Körperformen oder auch der Stimme, wird in Sekundenschnelle und i.d.R. unhinterfragt auf Subjektpositionen geschlossen. Es wird «erkannt», wer oder was das jeweilige Gegenüber «ist». Ebenso werden mittels spezifischer Raummerkmale Räume «gelesen» und eingeordnet. Solche Merkmale können der bauliche Zustand von Gebäuden, die Ausstattung mit spezifischen Dienstleistungsangeboten oder auch Gerüche und Geräusche sein. Dazu gesellt sich die An- oder Abwesenheit bestimmter Personen, die wiederum über ihren Körper erkannt und eingeordnet werden. Somit ergeben sich assoziative Verkettungen zwischen Körpern, Subjekten und Räumen.

Erzeugt werden Bedeutungen aber nicht erst in direkter Interaktion, sondern vornehmlich in Diskursen, die in gesellschaftlichen Institutionen und Praktiken verankert sind. Körper und Räume in ihrer Reflexivität werden diskursiv hervorgebracht und dabei symbolisch besetzt: Großwohnsiedlungen werden zu «Armenoder Ausländerghettos», Unterführungen oder dunkle Parks zu «Angsträumen», Bahnhöfe, an denen sich
Obdachlose oder DrogenkonsumentInnen aufhalten, zu «wahre(n) Pestbeulen» (von Gerkan 1997: 41) der Stadt.

Derartige Zuordnungen werden kaum reflektiert, geschweige denn als gesellschaftlich konstruiert in Frage gestellt. Vielmehr erscheinen sie als quasi naturgegeben. Gesellschaftlich dominante Bezeichnungen und Kategorien und dadurch geprägte routinisierte Erfahrungen schreiben sich in unser Bewußtsein ein. Die Bezeichnung setzt sich an die Stelle des Bezeichneten:

«Was uns lange genug vorgemacht wird, machen wir irgendwann nach bzw. betrachten es als «natürlich`, offensichtlich, als nicht zu hinterfragende Wahrheiten» (GENAZINO 1999).

Bedeutungszuschreibungen und Kategorisierungen sind eine wesentliche Grundlage unserer Orientierung und konstitutiv für soziales Leben. Von großer Bedeutung sind hier kulturell geteilte, symbolische Zeichen und Codes, auf die im alltäglichen Handeln zurückgegriffen wird. Sie sind eine Basis intersubjektiven Verstehens und ermöglichen zwischenmenschliche Interaktion. Normen, Codes und Kategorisierungen prägen aber auch unser Denken, Fühlen, Verhalten und Handeln; sie beeinflussen unseren Umgang mit Personen und Räumen.

So werden Räume, Subjekte und Körper fortwährend konstituiert. Mit dem Zurückgreifen auf Kategorien und auf äußerliche Merkmale, die diesen scheinbar natürlich zugrunde liegen, geht eine Wiederholung und Zementierung von Normen einher. Subjektpositionen und Hierarchisierungen werden so fortlaufend festgeschrieben und reproduziert. Verbunden ist dies mit gesellschaftlichen Ein- und Ausschlusspraktiken sowie mit einer Regulation von Raum und dessen Aneignungsmöglichkeiten. Bestimmten Personen und sozialen Gruppen werden Räume zugewiesen oder verwehrt. Körper und Raum erweisen sich also auch als Orte der Normierung und Disziplinierung (VILLA 1996).

Die diskursive Produktion von Körper und Raum hat aber nicht nur Effekte darauf, wie Körper und Räume «der Anderen» gelesen und gedeutet werden. Durch den Sachverhalt, daß wir alle unseren Körper «als verläßliche Verankerung unserer Identität erleben» (vILLA 1996: 147), prägt sie ebenso unser SelbstVerständnis und unsere Eigen-Wahrnehmung.

\section{Selbst-Verständliche Körper: Eigenwahrnehmungen}

Nachdem wir das Offen-Sichtliche neu beleuchtet haben, wollen wir auch noch das Selbst-Verständliche durcheinander bringen. 
Es geht bei der Betrachtung der Eigenwahrnehmung um das Verständnis des «Selbst». In der theoretischen Einführung wurde deutlich, daß der Körper eine große Bedeutung bei der Entstehung von Subjektivität hat. Dieser Prozeß wird auch «verkörperte Subjektivität» - embodied subjectivity - genannt. Verkörperte Subjektivität umfaßt nicht nur die materielle Körperlichkeit, sondern auch den imaginierten Körper (McDoWELL 1999: 39).

Bei der analytischen Trennung von Eigen- und Fremdwahrnehmung ist $\mathrm{zu}$ beachten, daß wir nicht in einen Körper hineineingeboren werden, sondern daß die Außenwelt einen Anteil an der Ausformung und Repräsentation unserer Körper hat. Die Wahrnehmung des eigenen Körpers ist stets von «äußeren» Wissensbeständen geprägt. Häufig spiegeln sich diese Wissensbestände in der Eigenwahrnehmung wider (Young 1989). Die Eigenwahrnehmung entspricht hier einer Folie, auf der einverleibte Strukturen «wahrheitsgetreu» abgebildet werden.

Jede Gesellschaft ist von raumkonstituierenden Diskursen geprägt. Die Macht dieser Diskurse liegt in ihrer naturalisierenden, objektivierenden Funktion und bewirkt, daß von einer Übereinstimmung von Fremd- und Eigenwahrnehmung ausgegangen wird. Nicht kompatible Eigenwahrnehmungen werden für Außenstehende, wie es auch ForscherInnen sind, meist erst durch ein Sichtbarmachen durch die Individuen selbst erkannt.

An dieser Stelle möchten wir beispielhaft das Zusammenwirken von Konstruktion und Normierung von Körpern und der Konstituierung und Aneignung von Räumen aufzeigen. Deutlich werden diese Prozesse durch die Betrachtung von körperlichen Verfassungen, die von der Norm abweichen. «Abweichende Körper» - deviant bodies - kann es nur geben, wenn es auch «normale» Körper gibt.

Als «normal» werden gesund aussehende Körper betrachtet, abweichend sind sichtbar kranke oder irgendwie nicht der vereinbarten Norm entsprechende Körper. Diese Einteilung ist ein gesellschaftliches Konstrukt, das durch Leitideale vorgegeben und durch individuelle Anpassung (oder Abwehr) etwa mittels Diät- und Fitness-Regimes sowie Kleider- und Körperleitbilder verkörpert wird.

Wenn wir uns Menschen ansehen, die mit lebensbedrohlichen Langzeitkrankheiten leben, merken wir, was mit Verkörperung gemeint ist. Verschiedene Strategien sind notwendig, um in der bestehenden für Normalbefähigte gestalteten Umgebung zurechtzukommen (McDowell 1999:39). Dadurch konstruieren Menschen mit «abweichenden Körpern» unterschiedliche Verständnisse von sich selbst als verkörperte Subjekte. Eine übliche Strategie von Menschen, die mit chronischen Krankheiten leben müssen, ist das Verschweigen der Krankheit und das Nicht-Auffallen wollen. Auch wenn es große Strapazen bedeutet, ver- suchen Kranke meist, dem Verhalten ihrer Mitmenschen zu entsprechen. Dadurch wird die Konstruktion des «gesunden» Körpers als der «normale» reproduziert. Diesem Verhalten liegt die Angst vor den stigmatisierenden Konsequenzen der Darstellung eines kranken Körpers zugrunde (Moss \& DYCK 1996). Als gesellschaftliche Prämisse gilt: Tabuisierung von Krankheit.

Aufschlußreich für diese Mechanismen ist der individuelle und gesellschaftliche Umgang mit lebensbedrohlichen chronischen Krankheiten. Ein prägnantes Beispiel für räumlich determinierte Körperstrategien ist das Leben von brustkrebskranken Frauen. Die Erkrankten durchlaufen während ihrer Krankheit einen Prozeß, der sich in verschiedenen Formen von Verkörperungen äußert.

Frauen erkranken in der Regel im Alter zwischen 25 und 70 Jahren an Brustkrebs (RKI 1999). Die übliche Therapieform ist die Zerstörung des Krebsgeschwüres. Die damit verbundene äußerlich sichtbare körperliche Veränderung ist meist die Entfernung der betroffenen Brust. Verbunden ist dieser Eingriff mit körperlichen Bewegungseinschränkungen, Todesangst und den psychosomatischen Folgen der medizinischen Behandlung (BISCHOFf 1999: 61). In der BRD erkranken jährlich über 45.000 Frauen an Brustkrebs, jede zehnte Frau ist im Laufe ihres Lebens davon betroffen. Also eine durchaus sichtbare Erkrankung, die aber dennoch in unserer Gesellschaft kaum visuell wahrnehmbar ist. Brustkrebs gehört zu den stark tabuisierten Krankheiten, die nicht sichtbar sein sollen. Der weiblichen Brust wird das Potential eines Sexsymbols zugeschrieben. Gleichzeitig symbolisiert sie Wärme, Geborgenheit und Nahrung. All diese Zuschreibungen an den weiblichen Körper gehen gemeinsam mit der Brust verloren. Mit einer Brustkrebsamputation wird die Entweiblichung des Körpers einer Frau assoziiert.

Bewältigungsstrategien der meisten Brustkrebskranken orientieren sich am näheren Umfeld. Die öffentliche Konfrontation mit der Krankheit fehlt fast völlig, die fehlenden Brüste bleiben versteckt. Die Strategie, mit dem abweichenden Körper umzugehen, hat Konsequenzen für die Raumaneignung. Ein diskursiv vermitteltes Wissen um die «Unzulänglichkeit» des eigenen, «entweiblichten» Körpers und die Nichtzugehörigkeit zur Welt der Schönen und Gesunden, machen es schwer, sich alltägliche Räume anzueignen, sich den eigenen Wünschen und Bedürfnissen entsprechend zu bewegen und sich der Persönlichkeit entsprechend sichtbar zu machen. Entsprechend ist der offensive Umgang mit einer positiv besetzten Präsentation einer Brustkrebserkrankung und damit verbundener Raumaneignung bisher eine Ausnahmestrategie (BISCHOFF 1999). Diese Nichtsichtbarkeit von bestimmten Körpern führt zu einer spezifischen Raumkonstruktion. 


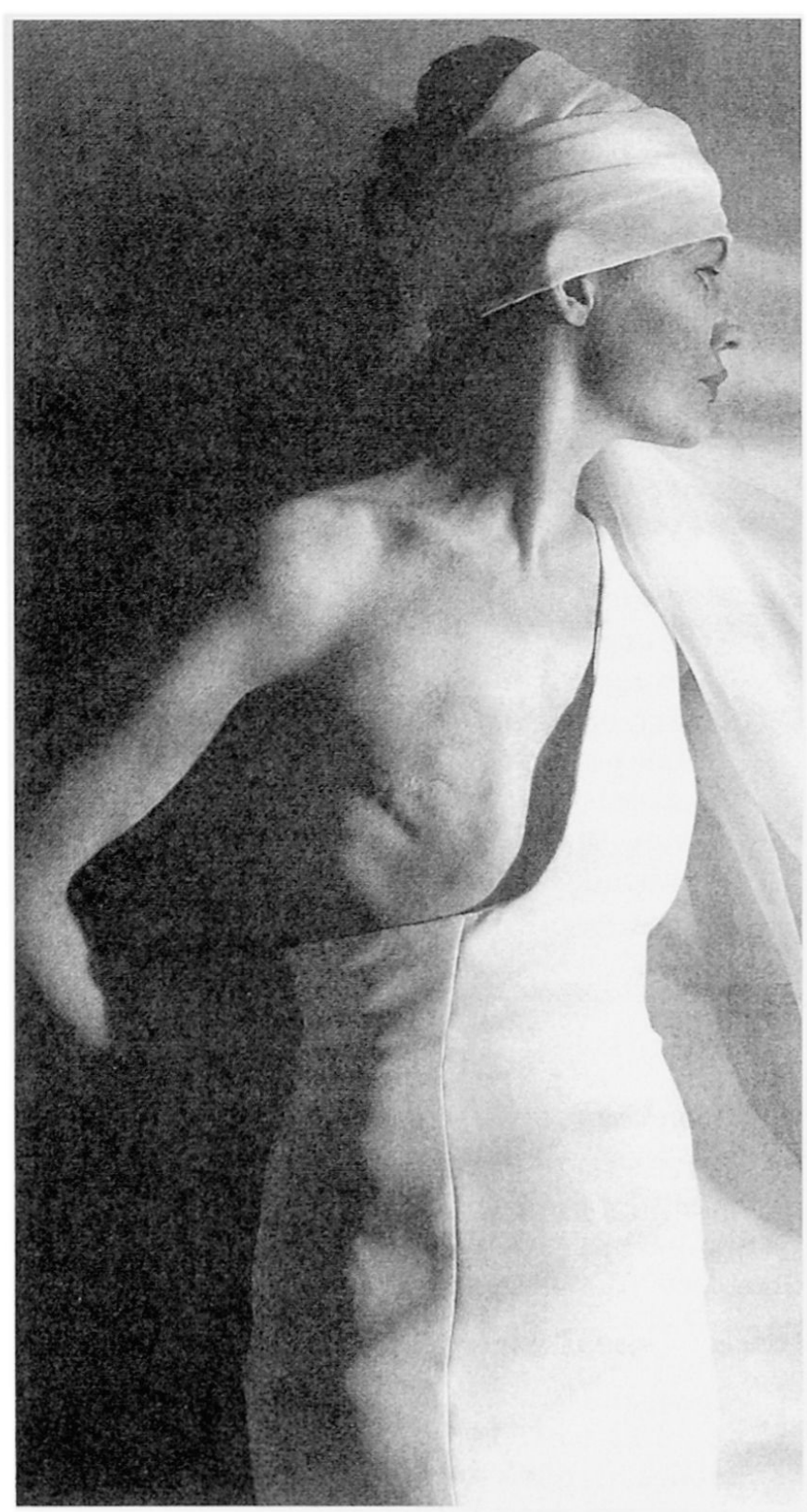

Abb. 1: New York Times Magazine, 15. August 1993, Titelseite (Nachdruck mit freundlicher Bewilligung) New York Times Magazine, August 15, 1993, frontpage (reprinted with permission)

New York Times Magazine, 15 août 1993, page de titre (avec l'autorisation de l'auteur)

Welche Neuverhandlung von Raumkonstruktionen durch die öffentliche Darstellung einer fehlenden Brust in Gang gesetzt wird, zeigte 1993 die US-amerikanische Künstlerin Matuschka. Sie hat sich mit ihrer entfernten Brust fotografiert und erreichte damit als Covergirl auf dem New York Times Magazine die Aufmerksamkeit von rund 30 Millionen Menschen. Obwohl ihre nicht vorhandene Brust sozusagen ins Auge sticht, hat sie eine sehr ästhetische weibliche Dar- stellungsform gewählt und sich so entgegen dem Entweiblichungsmodell inszeniert. Sie nannte ihr Porträt mit halb entblößtem Oberkörper, das sie nach der Amputation der rechten Brust zeigt, «Beauty Out of Damage». Körperverortung und Körpermacht werden hier in eine veränderte Beziehung gebracht. Je sichtbarer brustamputierte Frauen an verschiedenen Orten sind, desto weniger fehl am Platze wirken sie (BELL \& VAlentine 1995: 26).

Matuschkas Darstellung war lange Zeit eine einmalige öffentliche Artikulation. Am Tag nach unserem Vortrag, aus dem dieser Artikel hervorgegangen ist, erschien im Wochenmagazin STERN eine Titelgeschichte zum Thema Brustkrebs.

«50 betroffene Frauen bekennen sich (...) zu ihrem häufig verschwiegenen Leiden und erzählen von seinen Auswirkungen auf den Körper, auf die Seele und auf das Leben..."

und ließen sich mit ihrer Brustkrebserkrankung fotographieren (Neuhauser \& HaAse 1999: 28). Dieser Artikel und andere erschienen im Rahmen des «Internationalen Brustkrebsmonats», den die Weltgesundheitsorganisation (WHO) für den Oktober 1999 ausgerufen hatte. Die Wirkungen dieser und ähnlicher Präsentationen wären noch zu untersuchen.

Krankheit in der Öffentlichkeit sichtbar zu machen, ist eine Möglichkeit der Raumaneignung. Räume werden durch diese Strategie neu besetzt. Die individuelle Körperbewußtheit entscheidet jeweils darüber, in welcher Weise mit gesellschaftlichen Praktiken umgegangen wird. Der Körper ist dabei immer beides: ein Ort der Disziplinierung und ein Ort des Widerstandes (Foucault 1977, 1987).

\section{Fazit: «Verräumte» Körper - verkörperte Räume}

Die analytische Trennung von Fremd- und Eigenwahrnehmung haben wir aus zwei Gründen vorgenommen: Zum einen haben wir dadurch die Konstruiertheit von Körpern, Subjekten und Räumen verdeutlicht. Zum anderen sind die konstitutiven Bedeutungen von Körpern für Räume bewußt geworden.

Eine analytische Trennung ist wunderbar, allerdings darf nicht vergessen werden, die Ebenen wieder zusammenzuführen. Denn Eigen- und Fremdwahrnehmung sind wechselseitig aufeinander bezogen und können deswegen nicht unabhängig voneinander betrachtet werden. Beide sind geprägt durch gesellschaftliche Machtverhältnisse und für diese wiederum konstitutiv.

Um eine Dekonstruktion überhaupt durchführen zu können, ist diese Trennung sinnvoll, da sonst die Konstruktionsbedingungen und -folgen zu komplex und kaum sichtbar sind. Deutlich wurde:

Subjekte, Körper und Räume sind Orte der Disziplinierung und des Widerstandes. Sie sind Orte, 
an denen gesellschaftliche Strukturen reproduziert werden. Zugleich können Körper diese Strukturen aber auch verändern.

Poststrukturalistische Ansätze lassen den Körper als vermittelnde Instanz zwischen gesellschaftlichen MakroStrukturen (Kategorien wie Geschlecht, Ethnizität, Klasse) und subjektiver Identität auf der Mikro-Ebene verstehen. Der Körper ist der Ort, an dem sich die gesellschaftlichen Mikropraktiken mit der Organisation von Macht in großem Maßstab verbinden und er steht damit unmittelbar im Felde des Politischen (FouCAULT 1976).

Poststrukturalistische Ansätze bieten in diesem Zusammenhang Potentiale für die Analyse und das Verstehen der Logiken der Konstruktionsmechanismen. Sie ermöglichen eine Bewußtwerdung und Bewußtmachung der Konstruktionsprozesse und Produktionsbedingungen von Körpern, Subjekten und Räumen. Explizit feministisch poststrukturalistische Ansätze eröffnen die Chance, geschlechtsspezifische Hierarchien aufzubrechen und aus festen Zuschreibungen auf Körper und Räume auszubrechen.

Auf der Ebene der gesellschaftlichen Praxis entstehen damit Möglichkeiten, emanzipative Strategien zu entwickeln, wie Denaturalisierung, Sichtbarmachung und neue Formen der Raumaneignung. Damit sind Individuen in ihrer Vielfalt oder gesellschaftliche Gruppen in der Lage, sich solche Räume anzueignen und zu nutzen, die ihnen bisher verschlossen waren.

Durch die Einbeziehung von Körpern in geographische Raumkonzeptionen eröffnet sich eine neue Betrachtungs- und Analyseebene. Sie ist wesentlich, um die Organisation und wechselseitige Konstituiertheit räumlicher und gesellschaftlicher Verhältnisse und Prozesse verstehen und interpretieren zu können.

\section{Dank}

Dieser Artikel ist von der Konzeptionierung bis zur Endfassung eine Gemeinschaftsarbeit der Autorinnen. Die Idee entstand bei einem Workshop der AG SPUNK - Arbeitsgruppe Poststrukturalismus im Arbeitskreis Feministische Geographie - und wurde im Sommer 1999 bei einem mehrtägigen Treffen weiterentwickelt. Wir danken Sabine Malecek für anregende Beiträge.

\section{Literatur}

Bell, D. \& G. VAlentine (eds) (1995): Mapping Desire: Geographies of Sexualities. - London: Routledge.

BischOFF, K. (1999): Krebsgang! Zwei Schritte vor, einen zurück. - Zürich: Haffmanns Sachbuch Verlag. Bourdieu, P. (1982): Die feinen Unterschiede. Kritik der gesellschaftlichen Urteilskraft. - Frankfurt am Main: Suhrkamp.

Bourdieu, P. (1991): Physischer, sozialer und angeeig- neter physischer Raum. - In: WENTZ, M. (Hrsg.): StadtRäume. - Frankfurt am Main: 25-34.

BUTLER, J. (1997): Körper von Gewicht. - Frankfurt am Main: Suhrkamp.

Butler, J. (1998): Hass spricht. Zur Politik des Performativen. - Berlin: Berlin Verlag.

Derrida, J. (1976): Die différance. - In: Ders.: Randgänge der Philosophie. - Frankfurt am Main: 6-37.

Foucault, M. (1976): Überwachen und Strafen. Frankfurt am Main: Suhrkamp.

Foucault, M. (1977): Der Wille zum Wissen. Sexualität und Wahrheit I. - Frankfurt am Main: Suhrkamp.

Foucault, M. (1978): Dispositive der Macht: über Sexualität, Wissen und Wahrheit. - Berlin: Merve.

Foucault, M. (1987): Das Subjekt und die Macht. In: Dreyfus, H.L. \& P. Rabinow: Michel Foucault. Jenseits von Strukturalismus und Hermeneutik. - Frankfurt am Main: 243-261.

Genazino, W. (1999): Fühlen Sie sich alarmiert. - In: FRANKFURTER RUNDSCHAU vom 21.08.1999: ZB 3.

Gerkan, M. v. (1997): Renaissance der Bahnhöfe als Nukleus des Städtebaus. - In: Bund Deutscher ArCHItekten; Deutsche Bahn AG \& Förderverein DeutSChes ArChitektur Zentrum (Hrsg.): Renaissance der Bahnhöfe. Die Stadt im 21. Jahrhundert. - Berlin: 17-63.

List, E. (1994): Wissende Körper - Wissenskörper Maschinenkörper. - In: Die Philosophin 5: 9-26.

McDowelL, L. (1999): In and Out of Place: Bodies and Embodiment. - In: Dies.: Gender, Identity and Places. - Cambridge: 34-70.

Moss, P. \& I. DYcK (1996): Inquiry into environment and body: women, work and chronic illness. - In: Environment and Planning D: Society and Space 14: 737-53.

Neuhauser, U. \& E. HaAse (1999): Brustkrebs. Mutige Frauen berichten, wie sie ihr Leben meistern. - In: STERN 41: 26-38.

RoberT-KoCH-INSTITUT, RKI (1999): Neue Schätzungen der Gesamtzahl an Krebs Erkankender. - InternetVeröffentlichung: www.rki.de.

VILLA, P.-I. (1996): Spürbare Zugehörigkeiten. Klasse und Geschlecht als zweifache Positionierung des Leibes. - In: Fischer, U.L. et al. (Hrsg.): Kategorie: Geschlecht? Empirische Analysen und feministische Theorien. - Opladen: 141-162.

WeEdon, C. (1990): Wissen und Erfahrung. Feministische Praxis und poststrukturalistische Theorie. - Dortmund, Zürich: eFeF.

YounG, I.M. (1989): Throwing like a girl. - In: Allen, J. \& I.M. Young (eds): The Thinking Muse: Feminism and Modern French Philosophy. - Bloomington: 250-274. 
Zusammenfassung: Verkörperte Räume - «verräumte» Körper. Zu einem feministisch-poststrukturalistischen Verständnis der Wechselwirkungen von Körper und Raum

Menschliche Körper und Räume sind wechselseitig aufeinander bezogen und sehr ähnlichen gesellschaftlichen Konstitutionsbedingungen unterworfen. Dessen ungeachtet wurde der Körper mit seinen Bedeutungen für die Konstruktion und Aneignung von Räumen in der Geographie bisher kaum thematisiert. Dieser Beitrag widmet sich aus feministisch-poststrukturalistischer Perspektive dem dynamischen Wechselverhältnis von Körpern und Räumen. Besonders hervorgehoben werden dabei die Bedeutungen von Fremd- und Eigenwahrnehmung für vielfältige Raumaneignungsstrategien. Damit wird zugleich ein theoretischer Ansatz zur Diskussion gestellt, der neue Betrachtungs- und Analyseebenen eröffnet.

Summary: Bodied Spaces - «Boxed Bodies». For a feminist poststructuralistic understanding of interaction of body and space

Human bodies and spaces are interrelated with each other and underly very similar conditions of constitution. Nevertheless bodies and their importance for the construction and adoption of space have hardly been dealt with in geography. In this contribution we want to illuminate the subject from a feminist poststructuralist perspective. Of particular importance here are the role of outer and self perception for manifold strategies to take hold of space. The strategies that women who live with breast cancer have developed to perceive and act/react in their environment form the basis of a theoretical discussion on the topic of bodies and space. The discussion opens up new levels of reflection and analysis.

Résumé: Espaces incorporés - Corps «espacés». Pour une compréhension féministe et poststructuraliste des relations réciproques entre le corps et l'espace

Les corps humains et les espaces sont liés les uns aux autres et sont soumis à des conditions sociales de constitution très semblables. Cependant, la géographie n'a pas encore vraiment étudié le corps et son importance dans la construction et l'appropriation des espaces.
Cette contribution est centrée sur les corrélations dynamiques entre les corps et les espaces dans une perspective féministe et poststructuraliste. Elle met en évidence l'importance de la perception propre et extérieure dans les diverses stratégies d'appropriation de l'espace. En même temps, cet article présente une approche théorique qui ouvre de nouveaux niveaux de perspective et d'analyse.

Sybille Bauriedl, Institut für Geographie, Universität Hamburg, Bundesstr. 55, D-20146 Hamburg.

e-mail: bauriedl@geowiss.uni-hamburg.de

Katharina Fleischmann, Institut für Geographische Wissenschaften, Freie Universität Berlin, Malteserstr. 74-100, D-12249 Berlin.

Email:kathi@geog.fu-berlin.de

Anke Strüver, Department of Human Geography, Faculty of Policy Sciences, University of Nijmegen, P.O. Box 9108, NL-6500HK Nijmegen.

e-mail: a.struver@bw.kun.nl

Claudia Wucherpfennig, Fachbereich Geowissenschaften / Geographie, Johann Wolfgang Goethe-Universität Frankfurt am Main, Schumannstr. 58, D-60054 Frankfurt am Main.

e-mail:Wucherpfennig@em.uni-frankfurt.de 